\title{
Supplier's R\&D Investment in Manufacturer in A Decentralized Supply Chain with Quality and Price Sensitive Demand
}

\author{
Hengyun ZHANG* \\ School of Management and Economics, University of \\ Electronic Science and Technology of China Chengdu, \\ China \\ Department of Information Engineering, Sichuan Staff \\ University of Science and Technology, Chengdu, China \\ e-mail: zhyuestc@163.com \\ *Corresponding author
}

\author{
Ruoyu LU \\ School of Management and Economics, University of \\ Electronic Science and Technology of China Chengdu, \\ China \\ e-mail: lury@uestc.edu.cn
}

\begin{abstract}
This paper considers a supply chain with an upstream supplier and a downstream manufacturer, who faces a quality and price sensitive market demand. The supplier makes $R \& D$ investment in the manufacturer, which can lead to an improvement in the manufacturer's product quality, and hence, improve the efficiency of the entire supply chain. First, we derive the optimal retail price for the manufacturer, under any given $R \& D$ investment strategy of the supplier. Then, we characterize the supplier's optimal R\&D investment strategy. We show that both the supplier and the manufacturer can benefit from such an investment, under certain condition.
\end{abstract} theory

Keywords-R\&D investment; Quality improvement; Game

\section{INTRODUCTION}

Production efficiency of a supply chain depends on $R \& D$ resources, etc. [10]. When $R \& D$ resources are invested, the supply chain becomes more efficient, and all members can benefit from the more efficient supply chain. Therefore, all chain members have incentives to invest in R\&D resources. However, resource-based theory shows that many firms do not have sufficient R\&D resources [5]. When the downstream manufacturers are lack of R\&D resources, their upstream suppliers may provide R\&D resources for them. For example, in Japan, it has become an increasingly common business practice that, the upstream suppliers invest $R \& D$ resources in their downstream manufacturers [8].

There have been some studies focusing on $R \& D$ investment in decentralized supply chains. The existing studies mainly assume that R\&D investment can lead to either cost reduction or quality improvement [11]. For example, [12] study R\&D investment in a one-to-one supply chain. [1, 2] consider $R \& D$ investment in a one-to-many supply chain. [3, 4] investigate $R \& D$ investment in a many-to-one supply chain. $[7,9]$ explore R\&D investment in a many-to-many supply chain. Although the above studies have investigated R\&D investments in decentralized supply chains from different perspectives, they all assume that $R \& D$ investment are conducted by the firms themselves, and do not explore the problem that $R \& D$ investments are conducted by the supply chain partners.

In addition, [6] consider an assembly supply chain, where the downstream manufacturer makes R\&D investment in the upstream suppliers. They assume that the investment from the manufacturer can reduce the production costs of the suppliers. However, they do not address the problem of the upstream supplier's R\&D investment in the downstream manufacturer.

In this paper, we consider a supply chain consisting of an upstream supplier and a downstream manufacturer. The manufacturer is lack of $R \& D$ resources, and the supplier makes R\&D investment in the manufacturer. The resources invested by the supplier can lead to an improvement in the manufacturer's product quality. We assume that the demand faced by the manufacturer is quality and price sensitive. Moreover, we assume that the supplier first chooses its R\&D investment strategy, and then the manufacturer sets its retail price. The optimal decisions are studied and managerial insights are discussed, under the above assumptions.

In summary, the key contributions of our paper are as follows:

(a) We establish a model to study the problem of the upstream supplier's R\&D investment in the downstream manufacturer, in a decentralized supply chain.

(b) We derive the optimal retail price for the downstream manufacturer, under any given R\&D investment of the upstream supplier.

(c) We characterize the upstream supplier's optimal R\&D investment strategy, and get some interesting managerial insights.

\section{THE MODEL}

Considers a decentralized supply chain with an upstream supplier and a downstream manufacturer. The supplier provides a component to the manufacturer with wholesale price $w$, and then the manufacturer transfers it to the final product and sells it at the retail price $p$.

The supplier considers investing R\&D resources in the manufacturer. The invested resources can lead to a quality improvement in the manufacturer's product. The value of $R \& D$ resources invested by the supplier is denoted by $s, 0 \leq s \leq \bar{s}$, where $\bar{s}$ is the maximum value of $R \& D$ resources that can be invested by the supplier. The quality of the product is denoted by $\Delta(s)$, which increases in $S$, implying that the more R\&D 
resources is invested, the higher quality of the product will be.

We suppose similar the demand for the manufacturer's product is quality and price sensitive, which is given by [13]:

$$
q(p, \Delta(s))=a-b p+\beta \Delta(s) .
$$

In Equation (1), $a$ is the potential intrinsic demand for the product, $b$ represents the demand sensitivity to the retail price $p$ of the product, and $\beta$ corresponds to the demand sensitivity to the product quality $\Delta(s)$.

Now, the profits of the supplier and the manufacturer can be written as follows, respectively:

$$
\begin{gathered}
\pi_{u}(s)=(w-c)(a-b p+\beta \Delta(s))-s, \\
\pi_{d}(p)=(p-w)(a-b p+\beta \Delta(s)) .
\end{gathered}
$$

The problem is to choose an investment level for the $R \& D$ resources, and a retail price for the final product. The decisions to be made by the two players fall the following two stages: In the first stage, the supplier decides the investment level for the R\&D resources; In the second stage, the manufacturer chooses the retail price for its product.

\section{EQUILIBRIUM DECISIONS IN A DECENTRALIZED SUPPLY CHAIN}

In the decentralized supply chain, the upstream supplier and the downstream manufacturer are two independent decision makers, and choose the strategies to maximize their own profits, respectively. We will derive the equilibrium strategies in a backward manner. That is, we first study the manufacturer's price decision, provided that the $R \& D$ investment strategy is given. Then, we consider the supplier's investment strategy.

From Equation (2), we can establish the following theorem.

Theorem 1. For any R\&D investment level chosen by the upstream supplier, the optimal retail price for the downstream manufacturer is given by

$$
p=\frac{a+b w+\beta \Delta(s)}{2 b} .
$$

Proof. It follows from Equation (3) that

$$
\frac{d \pi_{d}(p)}{d p}=(a-b p+\beta \Delta(s))-b(p-w)=a-2 b p+b w+\beta \Delta(s),
$$

$$
\frac{d^{2} \pi_{d}(p)}{d p^{2}}=-2 b .
$$

From the above Equation, we know that the manufacturer's profit function $\pi_{d}(p)$ is strictly concave in its retail price $p$. Thus, the optimal retail price for the manufacturer satisfies the first-order condition,

i.e.,

$$
\frac{d \pi_{d}(p)}{d p}=a-2 b p+b w+\beta \Delta(s)=0
$$

. Solving the

first-order condition for $p$, we can get Equation (4). We thus complete the proof of Theorem 1.

From Equation (4), we know that the retail price for the product increases as the wholesale price increases. This is not surprising, since a downstream manufacturer with a higher procurement cost (i.e., wholesale price) will set a higher retail price for its product. Second, because $\Delta(s)$

level $S$ increases, then we can also know from Equation (4) that the retail price increases as the investment level $S$ increases. That is, a higher retail price will be chosen by the downstream manufacturer for the product with a higher quality. This is rather intuitive.

Knowing that the downstream manufacturer sets the retail price according to Equation (4), the upstream supplier chooses a R\&D investment level to maximize its profit.

Substituting Equation (4) into Equation (2) and Equation (3), respectively, we can rewrite the supplier's profit function and the manufacturer's profit function, as follows, respectively:

$$
\begin{gathered}
\pi_{u}(s)=\frac{(w-c)(a-b w+\beta \Delta(s))}{2}-s, \\
\pi_{d}=\frac{(a-b w+\beta \Delta(s))^{2}}{4 b} .
\end{gathered}
$$

From Equation (5) and Equation (6), we have the following theorem.

Theorem 2. (i) The downstream manufacturer can always benefit from the upstream supplier's R\&D investment. (ii) The upstream supplier may not benefit from its R\&D investment.

Proof. Note that $\Delta(s)$ increases as $s$ increases, then we can get theorem 2(ii) from Equation (5); and obtain theorem 2(i) from Equation (6). We thus complete the proof of Theorem 2.

When the upstream supplier makes R\&D investment in the downstream manufacturer, the product quality of the manufacturer is improved, and thus, the entire supply chain becomes more efficient, which will benefit each 
player in such a supply chain. As a matter of fact, we can calculate from Equation (5) and Equation (6) that, the benefit caused by the R\&D investment for the supplier is

$$
\pi_{u}(s)-\pi_{u}(0)=\frac{(w-c) \beta[\Delta(s)-\Delta(0)]}{2},
$$

And the benefit caused by the R\&D investment for the manufacturer is

$$
\left.\pi_{d}\right|_{s=s}-\left.\pi_{d}\right|_{s=0}=\frac{(a-b w+\beta \Delta(s))^{2}-(a-b w+\beta \Delta(0))^{2}}{4 b} .
$$

Consequently, the downstream manufacturer always be willing to be invested. However, the upstream supplier will weigh up the benefit $\pi_{u}(s)-\pi_{u}(0)$ caused by the R\&D investment and the corresponding investment cost $S$, and may not be willing to invest. Specifically, if $\pi_{u}(s)-\pi_{u}(0)>s$, then the supplier should invest; and if $\pi_{u}(s)-\pi_{u}(0)<s$, then the supplier should not invest.

Next, we consider the problem of the upstream supplier's R\&D investment strategy. Recall that we suppose that the product quality $\Delta(s)$ increases in $s$. More specifically, we suppose that the product quality function is

$$
\Delta(s)=\Delta+\delta_{1} s+\delta_{2} s^{2}
$$

Where $\Delta$ is the intrinsic quality of the product with no $\mathrm{R} \& \mathrm{D}$ investment from the supplier, $\Delta \geq 0$; and $\delta_{1}$ and $\delta_{2}$ characterize quality sensitivity to the supplier's R\&D investment, i.e., the efficiency of quality improvement caused by the R\&D investment. To further characterize the product quality function, we consider the following three settings.

(i) $\delta_{1} \geq 0$ and $\delta_{2}=0$; (ii): $\delta_{1} \geq 0$ and $\delta_{2}>0$; (iii): $\delta_{1} \geq 0$ and $\delta_{2}<0$.

The first setting implies that the product quality $\Delta(s)$ is linearly increasing in R\&D investment level $s$; the second setting means that the product quality $\Delta(s)$ is convexly increasing in R\&D investment level $s$; the last setting indicates that the product quality $\Delta(s)$ is concavely increasing in R\&D investment level $s$. In addition, to ensure that $\Delta(s)$ always increases as $s$ increases, we require that $-\delta_{2} \leq \frac{\delta_{1}}{2 \bar{s}}$ in the third setting;
Otherwise, $\Delta(s)$ will decrease as $s$ increases in the third setting.

This allows us to obtain the following theorem.

Theorem 3. (i) In the first setting, if $\delta_{1}<\frac{2}{(w-c) \beta}$ , then the optimal R\&D investment strategy is $s^{*}=0$; if $\delta_{1}>\frac{2}{(w-c) \beta}$, then the optimal R\&D investment strategy for the supplier is $s^{*}=\bar{s}$.

(ii) In the second setting, if

$$
\delta_{1}+\delta_{2} \bar{s}<\frac{2}{(w-c) \beta}
$$

then the optimal R\&D investment strategy is $s^{*}=0$; if $\delta_{1}+\delta_{2} \bar{s}>\frac{2}{(w-c) \beta}$ investment strategy is $s^{*}=\bar{s}$.

(iii) In the third setting, if $\delta_{1}<\frac{2}{(w-c) \beta}$, then the optimal strategy is $s^{*}=0$; if $\delta_{1}>\frac{2}{(w-c) \beta}$ and $\delta_{1}+2 \delta_{2} \bar{s}>\frac{2}{(w-c) \beta}$ , then the optimal strategy is $s^{*}=\bar{s} \quad ; \quad$ if $\quad \delta_{1}>\frac{2}{(w-c) \beta} \quad$ and $\delta_{1}+2 \delta_{2} \bar{s}<\frac{2}{(w-c) \beta}$ $s^{*}=\frac{1}{\beta(w-c) \delta_{2}}-\frac{\delta_{1}}{2 \delta_{2}}$.

Proof. It follows from Equation (5) and Equation (7) that

$$
\frac{d \pi_{u}(s)}{d s}=\frac{\beta(w-c)\left(\delta_{1}+2 \delta_{2} s\right)}{2}-1,
$$

$$
\frac{d \pi_{u}(s)}{d s}=\beta(w-c) \delta_{2}
$$

Then, we know that:

(i) When $\delta_{1} \geq 0$ and $\delta_{2}=0$, then $\pi_{u}(s)$ is linear in $s$, and hence $\pi_{u}(s) \leq \max \left\{\pi_{u}(0), \pi_{u}(\bar{s})\right\}$ for 


$$
\begin{aligned}
& \text { any } 0 \leq s \leq \bar{s} . \quad \text { Solving } \pi_{u}(0)>\pi_{u}(\bar{s}) \text { and } \\
& \pi_{u}(0)<\pi_{u}(\bar{s}) \text {, respectively, we get Theorem 3(i). }
\end{aligned}
$$

(ii) When $\delta_{1} \geq 0$ and $\delta_{2}>0$, then $\pi_{u}(s)$ is convex in $s$. That is, $\pi_{u}(s) \leq \max \left\{\pi_{u}(0), \pi_{u}(\bar{s})\right\}$ for any $0 \leq s \leq \bar{s}$. Solving $\pi_{u}(0)>\pi_{u}(\bar{s})$ and $\pi_{u}(0)<\pi_{u}(\bar{s})$, respectively, we get Theorem 3(ii).

(iii) When $\delta_{1} \geq 0$ and $\delta_{2}<0$, then $\pi_{u}(s)$ is concave in $s$, i.e., $\frac{d \pi_{u}(s)}{d s}$ is decreasing in $s$. Firstly, if $\left.\frac{d \pi_{u}(s)}{d s}\right|_{s=0}<0$, then $\pi_{u}(s)$ achieves its maximum at $s^{*}=0$; Secondly, if $\left.\frac{d \pi_{u}(s)}{d s}\right|_{s=\bar{s}}>0$ then $\pi_{u}(s)$ achieves its maximum at $s^{*}=\bar{s}$. Finally, if $\left.\frac{d \pi_{u}(s)}{d s}\right|_{s=0}>0 \quad$ and $\left.\frac{d \pi_{u}(s)}{d s}\right|_{s=\bar{s}}<0$, then $\pi_{u}(s)$ achieves its maximum at $s^{*}$, which is uniquely determined by the first-order condition, i.e., $\frac{d \pi_{u}(s)}{d s}=0$

This enables us to get Theorem 3(iii). We thus complete the proof of Theorem 3.

Theorem 3 characterizes the upstream supplier's optimal R\&D investment strategy under each of the three settings, and states that the supplier's investment strategy highly depends on parameters $\delta_{1}$ and $\delta_{2}$. Specially, In the first setting, if the efficiency of quality improvement caused by the supplier's R\&D investment $\delta_{1}$ is relatively low, the supplier will not invest, i.e., $s^{*}=0$; if the efficiency of quality improvement caused by the supplier's R\&D investment $\delta_{1}$ is relatively high, the supplier will invest fully, i.e., $s^{*}=\bar{S}$. Similar analysis can be conducted for the second and the third settings. Our theorem reveals the following important insights: The supplier is more likely to makes $R \& D$ investment in the manufacturer, when the efficiency of quality improvement caused by the $R \& D$ investment is relatively high.

\section{SUMMARY}

Although there have many papers focusing on quality improvement investment (or cost reduction investment) in the supply chains, they mainly assume that the quality improvement (or cost reduction) is caused by the $R \& D$ investment by the firms themselves and ignore the possibility that $R \& D$ investments are conducted by their upstream partners. This paper considers the problem of the upstream supplier's quality improvement in the downstream manufacturer. The main results obtained in this paper are as follows:

(a) When the upstream supplier makes R\&D investment in the downstream manufacturer to improve its product quality, the retail price of the product increases;

(b) The supplier's $R \& D$ investment can always benefit the manufacturer, but may cause detriment to the supplier itself.

(c) The optimal R\&D investment strategy for the supplier highly depends on the efficiency of quality improvement caused by the R\&D investment.

It is interesting to consider the problem of R\&D investment between vertically related players in a supply chain with uncertain demand.

\section{ACKNOWLEDGEMENT}

This paper is supported by the National Natural Science Foundation of China (No. 70772068).

\section{REFERENCES}

[1] S. Banerjee, P. Lin, Vertical research joint ventures, International Journal of Industrial Organization. 19(2001) 285-302.

[2] S. Banerjee, P. Lin, Downstream R\&D, raising rivals' costs, and input price contracts, International Journal of Industrial Organization. 21(2003) 79-96.

[3] F. Bernstein, A.G. Kök, Dynamic cost reduction through process improvement in assembly networks, Management Science. 55(2009) 552-567.

[4] F. Bernstein, A.G. Kök, A. Meca, Cooperation in assembly systems: The role of knowledge sharing networks, European Journal of Operational Research. 240(2015) 160-171.

[5] T.K. Das, B.S. Teng, A resource-based theory of strategic alliances, Journal of management. 26(2000) 31-61.

[6] H. Fu, Y. Ma, X. Cai, Downstream firm's investment with equity holding in decentralized assembly systems, Omega. Forthcoming (2017).

[7] S. Gupta, Research note-channel structure with knowledge spillovers, Marketing Science. 27(2008) 247-261.

[8] L. Hollerman, Liberalization and Japanese trade in the 1970s, Asian Survey. 10(1970) 427-437.

[9] A. Ishii, Cooperative R\&D between vertically related firms with spillovers, International Journal of Industrial Organization. 22(2004) 1213-1235.

[10] J.B. Jin, C.S. Leem, C.H. Lee, Research issues and trends in industrial productivity over 44 years, International Journal of Production Research. 54(2016) 1273-1284.

[11] M. Leng, Z. Li, L. Liang, Implications for the role of retailers in quality assurance, Production and Operations Management. Forthcoming (2015).

[12] M. Usta, F. Erhun, W.H. Hausman, Supply licensing when the manufacturer strategically commits to invest in R\&D, Naval Research Logistics. 61(2014) 341-350.

[13] R.D. Banker, I. Khosla, K.K. Sinha, Quality and competition, Management Science. 44(1998) 1179-1192. 